\title{
Debris discs around stars: The 2004 ISO legacy*
}

\author{
Marie Jourdain de Muizon \\ Leiden Observatory, PO Box 9513, 2300 RA Leiden, The Netherlands, and LESIA, Paris \\ Observatory, 92190 Meudon, France
}

\begin{abstract}
Debris discs around stars were first discovered by IRAS, the Infrared Astronomical Satellite in 1983. For the first time material orbiting another star than the Sun, but distinct from a circumstellar envelope, was observed through its far infrared emission. This major discovery motivated astronomers to investigate those discs by further analysing the IRAS data, using ground-based telescopes for the hunting of exoplanets, developing several projects using ISO, the Infrared Space Observatory, and now exploiting IDA, the ISO Data Archive. This review presents the main ISO results, statistical as well as individual, on debris discs in orbit around pre-main-sequence and main-sequence stars.
\end{abstract}

Received: 3 August 2004, Accepted: 2 November 2004

\section{INTRODUCTION}

Discs around stars appear during the early stages of stellar evolution. About 4.6 Gyr ago, the Sun - like any other star - formed in a local contraction of an interstellar cloud of molecular gas and dust. During its first few million years the Sun was surrounded by a warm rotating disc of gas and dust that on one hand fed material onto the forming star and on the other hand led to the formation of comets, planets and planetesimals, asteroids and other objects. This kind of warm disc is also found around other pre-main-sequence stars, such as T Tauri stars, Herbig Ae/Be stars and ZAMS stars (Robberto et al. 1999). They contain some original interstellar dust, are optically thick and extend to a few AU. After a few $10^{7}$ years, the warm inner part of the solar disc was dissipated while a cooler debris disc remained in the outer part of the newly formed solar system. Such a disc contains mainly interplanetary dust resulting from collisions, is optically thin and extends up to a few hundred AU. It is such a debris disc that was first discovered by IRAS (Aumann et al. 1984) around Vega, $\alpha$ Lyr, one of the best calibrated and extensively used photometric standard in the visual range, then found to radiate more midand far-infrared emission than its photosphere can produce, and also around $\alpha$ PsA, $\varepsilon$ Eri and $\beta$ Pic. A systematic search for Vega-like discs in the IRAS survey led to several other identifications but was not very conclusive due to the limited sensitivity (Backman \& Paresce 1993, Plets and Vynckier 1999). As follow-up of IRAS, several surveys were undertaken with ISO (Kessler et

\footnotetext{
* Based on observations with ISO, an ESA project with instruments funded by ESA Member States (especially the PI countries: France, Germany, the Netherlands and the United Kingdom) and with the participation of ISAS and NASA.
}

(c) Manuscript - To be published by Springer in 2005.

Space Science Review ISO Special Issue. 
al. 1996; Kessler et al. 2003). The most unbiased was obtained by Habing et al. $(1999,2001)$ but others concentrated on stars in open clusters (Spangler et al. 2001), G dwarfs (Decin et al 2000), or selected MS stars (Fajardo-Acosta et al 1999). Several case studies on the most ISO observed discs are also presented: Vega and $\beta$ Pic (Heinrichsen et al. 1998, 1999), $\rho^{1}$ Cnc (Dominik et al. 1998), HD 207129 (Jourdain de Muizon et al. 1999), five Vega-like stars including $\alpha$ PsA (Fajardo-Acosta et al. 1997). Finally ISO spectroscopic observations evidenced the presence of amorphous and crystalline silicates, forsterite, PAHs (Meeus et al. 2001, Bouwman et al. 2001) and molecular hydrogen (Thi et al. 1999, 2001a, 2001b) in the discs of several pre-mainsequence stars. See also two more general reviews on the subject by Lagrange et al. (2000) and Zuckerman (2001).

\section{ISO surveys}

\subsection{GENERAL STATISTICS}

Habing et al. (1999, 2001) proposed to determine the incidence of Vega-like debris discs in a distance limited sample of main-sequence stars. The stars were carefully selected from Johnson \& Wright (1983) who computed farinfrared fluxes for $93 \%$ of the 2150 stars in the "Woolley catalog of stars within $25 \mathrm{pc}$ from the Sun". After rejecting all stars either too faint for ISO at $60 \mu \mathrm{m}$ or for which an infrared excess would be ambiguous to interpret as a disc, their final selection consisted of 84 main-sequence stars of spectral types from A to $\mathrm{K}$. Within this range, no spectral type was privileged. The stars were measured with ISO at 25, 60,90 and $170 \mu \mathrm{m}$ in a total observing time of 65 hours.

Based on ISOPHOT (Lemke et al., 1996; Laureijs et al., 2003) C100 $3 \times 3$ minimaps at $60 \mu \mathrm{m}$, Habing et al. $(1999,2001)$ found that $17 \%$ of the stars in their sample do have a disc. Lachaume et al. (1999) determined the age of the 84 stars. It appeared that all stars younger than 300 Myr have a disc, $70 \%$ of the stars younger than $400 \mathrm{Myr}$ still have a disc but this is the case for only $8 \%$ of the stars older than 1 Gyr. Thus it seems that most stars arrive on the main-sequence surrounded by a disc, and for the majority of them the disc is dissipated within a few hundred Myr (Figure 1). The decay of the discs is attributed to the destruction and escape of planetesimals; indeed the collision of planetesimals is a good source of dust, necessary to replenish the disc because dust particles disappear on a much shorter timescale relative to the lifetime of the discs. The timescale of the dissipation of the disc corresponds to the end of the heavy bombardment phase in the Solar System, which is identified by dating the cratering on the Moon, Mercury, Ganymede and Callisto. Craters are due to impacts from planetesimals and this bombardment appears to have 


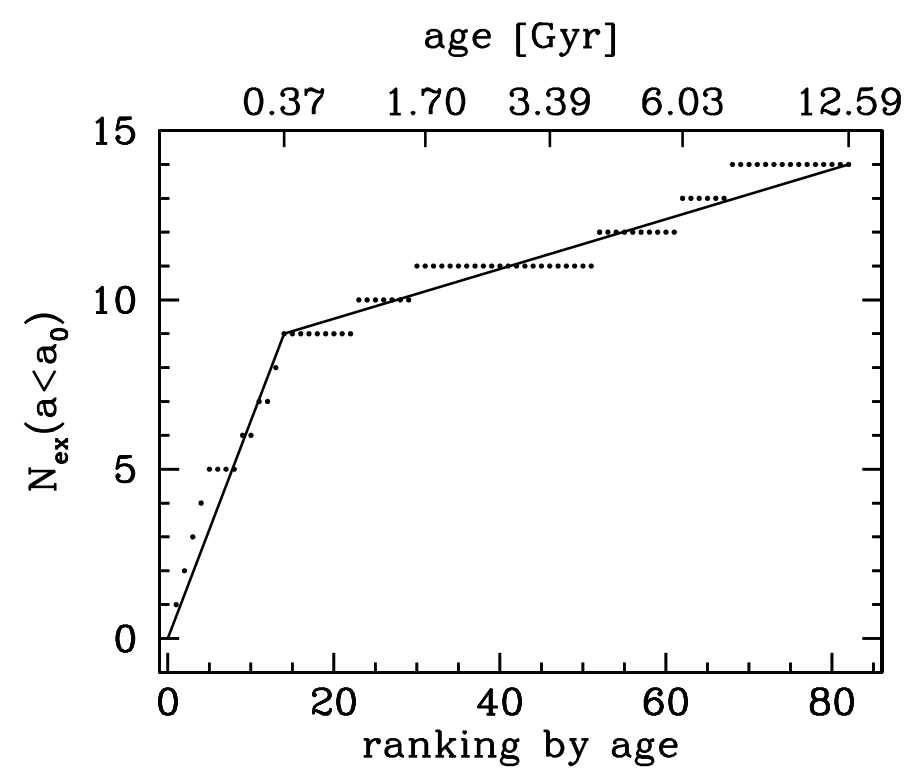

Figure 1. Cumulative distribution of stars with an infrared excess, as a function of the index, sorted by age. X-axis is rank at the bottom and age at the top of the figure. Y-axis is the number of stars up to that rank which have a disc. Each dot on the figure represents a star of the sample. For each star with an infrared excess, $\mathrm{N}_{\mathrm{ex}}$ increments by one. The two segments of a continuous straight line correspond to the perfect situation in which most debris discs are no longer detectable after $400 \mathrm{Myr}$ (from: Habing et al. 2001).

stopped about 4 Gyr ago (Shoemaker \& Shoemaker 1999, Morbidelli et al. 2001). Thus the timescale of $400 \mathrm{Myr}$ would not trace, properly speaking, the disappearance of the dust discs, but the termination of the production of dust by collisions of planetesimals, hence the drastic reduction in the occurence of these larger bodies (Jourdain de Muizon et al. 2001).

Using $25 \mu \mathrm{m}$ ISOPHOT data on 81 stars from the Habing et al. (2001) sample, Laureijs et al. (2002) found that 5 stars (6\%) have an infrared excess that can be attributed to a disc of dust temperature between 50 and $120 \mathrm{~K}$. The 5 stars are younger than $400 \mathrm{Myr}$, thus indicating that warm debris discs are relatively rare and concern the younger stars only. The survey confirms that there seems to be an absence of detectable amounts of dust close to the stars $(D \leq 20 \mathrm{AU})$. From a survey of 38 main-sequence stars using IRAS and ISOPHOT data, Fajardo-Acosta et al. (1999) found no star with a significant excess at $12 \mu \mathrm{m}$, and a fraction of $\sim 14 \%$ excess stars at $20 \mu \mathrm{m}$. However, this fraction is difficult to interpret since the ISOPHOT data in their study were inconclusive and the detections needed confirmation. The absence of $12 \mu \mathrm{m}$ excess indicates that the discs are not warmer than $200 \mathrm{~K}$. Finally Jourdain de Muizon \& Laureijs (2005) found 3 more excess stars at $60 \mu \mathrm{m}$ and one more at $90 \mu \mathrm{m}$ by exploring the ISOPHOT chopped data which had been obtained 
at an early stage in the ISO mission but were later replaced by minimaps (see Habing et al. 2001).

\subsection{G STARS}

Decin et al. (2000) studied the incidence of the Vega phenomenon around $\mathrm{G}$ dwarfs. The stars were selected from the CORALIE sample (planet-search programme around stars closer than $50 \mathrm{pc}$ and of spectral type from F8 to M1, excluding giants and faint cool dwarfs). Only southern stars were considered and the main selection criteria were observability and detectability by ISO at $60 \mu \mathrm{m}$. Confused systems such as multiple stars and those against a high cirrus background were rejected. Finally, this ISO programme consisted of 69 stars, 34 of which were effectively observed and results are given for 30 of them (ISOPHOT minimaps at $60 \mu \mathrm{m}$ ).

Of the $30 \mathrm{G}$ dwarfs in Decin et al. (2000), 5 (17\%) have a debris disc and 4 out of these 5 are older than 3 Gyr. This is in good agreement with Habing et al. (2001) who have $21 \mathrm{G}$ dwarfs in their sample and find than $4(19 \%)$ of them have a debris disc; 3 out of these 4 are older than 5 Gyr (they are part of their $8 \%$ stars older than $1 \mathrm{Gyr}$ and which still have their disc, see Sect. 2.1). However, 2 of the excess stars in Decin et al. (2000) have fractional luminosities of their disc comparable to the disc of $\beta$ Pic which was a unique case so far, whereas the others and all the discs around G stars in Habing et al. (2001) are between one and two orders of magnitude fainter. Also no correlation was found between the existence of a disc and a planet around the stars of the Decin et al. (2000) sample.

Thus for both groups of authors, about $18 \%$ of the $\mathrm{G}$ stars do have a disc and about $80 \%$ of these discs are around G stars older than $3 \mathrm{Gyr}$. Why do G stars appear to keep their discs longer than A, F or K stars is not yet understood. Around the Sun, the zodiacal light and the Kuiper Belt are probably some remnants of the earlier disc.

\subsection{STARS IN OPEN CLUSTERS}

Spangler et al. (2001) used ISOPHOT to observe a total of 148 stars, of which 87 young $(50-700 \mathrm{Myr})$, nearby $(\mathrm{d}<120 \mathrm{pc})$ main-sequence stars in open clusters ( $\alpha$ Persei, Coma Berenice, Hyades, Pleiades, UMa) of spectral type A to K, 41 T Tauri stars in the clouds Chamaeleon I, Scorpius and Taurus (d $\approx$ $140-150 \mathrm{pc})$, and a sample of 19 isolated young nearby field stars $(\mathrm{d}<60 \mathrm{pc})$ and another field star. They obtained ISOPHOT chopped observations with the $\mathrm{C} 100$ detector at 60 and $100 \mu \mathrm{m}$ or raster at 60 and $90 \mu \mathrm{m}$. The goal was to determine an evolutionary sequence for circumstellar disc characteristics, hence their choice of well-studied clusters in which stellar ages are fairly well defined. Although spectral types span the range A to K the authors gave a preference to solar-type stars and priviledged spectral types $F$ and $G$ in 
their star selection. They detected 36 stars, of which 33 show evidence for a far-infrared excess, i.e. $22 \%$ of their hole sample. More than one third were already thought to have an IR excess in their IRAS data, the rest are new ISO detections. These latter consist of 13 cluster stars, 5 young field stars and one other field star, with an ISO excess emission at 60,90 or $100 \mu \mathrm{m}$. Among the main-sequence excess stars, spectral type F clearly dominates. Given the sample size of the statistics, the $22 \%$ excess stars in Spangler et al. (2001) is not too far from the 17\% in Habing et al. (2001) or in Decin et al. (2000). The difference is most likely due to the selection criteria, the sample of Habing et al. (2001) being the most unbiased and homogeneous. Discs around T Tauri stars are closer to accretion discs (optically thick) and are not properly called debris discs (optically thin) like those around main-sequence stars. We only mention them in this ISO review because they are precursors of the debris discs but they cannot be treated equally when defining the incidence of debris discs.

A convenient parameter to describe systems exhibiting excess emission from circumstellar discs is the fractional excess luminosity, $f_{d}=L_{e x} / L_{\star}$, where $L_{e x}$ is the luminosity of dust and $L_{\star}$ is the stellar bolometric luminosity. Although $L_{e x}$ is not easy to estimate as it requires to know the complete infrared spectrum of the excess, it can be done approximately using both the IRAS and ISO data. A very interesting result of Spangler et al. (2001) is how the IR excess evolves with time. They established that the fractional excess luminosity $f_{d}$ decreases with stellar age according to the power law $f_{d} \propto(\text { age })^{-1.76}$. This is compatible with a collisionally replenished disc as suggested in Habing et al. (2001). Spangler et al. (2001) claim they do not see evidence for an abrupt cessation of the debris disc phenomenon as reported in Habing et al. (2001). It is indeed not obvious from their Figure 1, but in fact 27 out of their 33 excess stars (82\%) are younger than $400 \mathrm{Myr}$, and the other 6 are between 400 and 625 Myr. This is in perfect agreement with Habing et al. (2001). It is clear that debris discs are mostly found around young stars. Spangler et al.'s sample is biased towards young stars anyway. All excess stars in their sample are younger than the end of the late heavy bombardment on the Moon.

Robberto et al. (1999) present preliminary results on 97 very young stars in 5 open clusters, 3 of which are in common with those in Spangler et al. (2001), namely Chamaeleon, $\alpha$ Per and Pleiades, and they are all younger than 300 Myr. Using ISOPHOT with the C100 detector at $60 \mu \mathrm{m}$ and P2 detector at $25 \mu \mathrm{m}$ they detected only 4 stars, i.e $4.1 \%$ of their hole sample. The 4 stars detected are all $\mathrm{T}$ Tauri stars in the Chamaeleon I cluster; 3 of them are classical $\mathrm{T}$ Tauri stars which are still in the accretion phase (there are only 6 such stars known in this cluster) and the fourth one is probably in the transition phase. Their results show that the transition from an optically 
thick disc (or accretion disc) to an optically thin one (or debris disc) occurs on a timescale of $\sim 10 \mathrm{Myr}$, with a transition phase lasting less than $\sim 0.3 \mathrm{Myr}$.

\subsection{RE-ANALYSIS OF THE ABOVE SURVEYS}

Greaves and Wyatt (2003) have addressed the issue of the incidence of debris discs among A- and G- type main- sequence stars from the various published ISO surveys and some IRAS discs (about 200 stars). Their analysis shows a much higher detection rate of debris disks towards A stars (9/20 hence 45\%) than $\mathrm{G}$ stars (11/180 hence 6\%), and this big difference in disc detection rate applies even when stars of similar age are considered. From the observations, disc lifetime is estimated to about $0.5 \mathrm{Gyr}$ and may occur at any time during the main-sequence life of the star. Disc lifetime and high occurrence of discs among A stars were two conclusions already reached by Habing et al. (2001), but they are now also verified on a much larger sample. The fact that A stars have a much shorter lifetime and a larger disc mass than G-stars, implies the disc lifetime is a much higher fraction of the star lifetime in A stars than in Gstars and thus it is not surprising that A stars discs are more often seen than in $\mathrm{G}$ stars and also often seen in the first half of the A star lifetime (i.e. younger than $500 \mathrm{Myr}$ ). The disc mass scans a range of about 100, decline with time is slow and follows a power law not steeper than $t^{-1 / 2}$. The conclusion that the debris disc lifetime can take place at any time during the main-sequence, and not necessarily at the beginning of the stellar life, would account for the existence of a few detected debris disks around some old G stars. According to the models (Kenyon \& Bromley 2002a, 2002b), perturbing planetesimals can form slowly at large orbital radii as late as a few Gyr, thus producing dust by collisions and replenishing a disc, but the same models cannot really explain the more or less constant disc duration of about $0.5 \mathrm{Gyr}$. Also based on the various ISO surveys, the age dependence of the Vega phenomenon has been studied observationally and theoretically respectively by Decin et al. (2003) and Dominik and Decin (2003). Decin et al. (2003) have critically reexamined the stellar age estimates from the existing ISO surveys. Two aspects are to be considered: i) the time dependence of the disc dust mass, measured by $f_{d}$ the fractional (i.e. disc/star) luminosity and ii) the incidence of debris discs versus stellar age. Decin et al. (2003) came to the conclusion that there is no clear trend in the time dependence of $f_{d}$, in particular no power-law with an index of $\approx-2$ (e.g. Spangler et al. 2001), but rather a large spread irrespective of stellar age. However, for a given stellar age, the maximum $f_{d}$ is about $10^{-3}$ (upper cut-off). They also identify a few cases of very young stars with intermediate or small excess (i.e. below the lower cut-off for $f_{d}$ which is about $10^{-5}$ ), and they conclude that Vega-like stars are more common in young stars that in old stars, a conclusion which had already been reached by Habing et al. (1999). The results of Decin et al. (2003) are in agreement 
with Habing et al. (1999, 2001) who had the most unbiased sample of the ISO Vega-like studies (see Sect. 2.1). They differ significantly from those of Spangler et al. (2001), but that could be explained because the latter studied mainly stars in clusters, thus of similar age and evolution pattern, hence the rather steep power law they could derive for $f_{d}$ versus time. Dominik and Decin (2003) have tried to provide a coherent theoretical picture of the various ISO observations of debris discs. On one hand, without replenishment, the dust in a debris disc should disappear in few thousands years under the Poynting-Robertson effect, i.e. a drag on interplanetary particles caused by their interaction with solar (respectively stellar) radiation, which causes the particles to lose orbital momentum and spiral into the Sun (respectively star). On the other hand the discs last much longer than a few thousands years and some of them are seen in old stars, thus the dust must be replenished. The model must account for both the existence of the disc at a given stage of stellar evolution and also the disc evolution (from its formation to its dissipation). The former needs an accurate knowledge of stellar ages, the latter an accurate estimate of $f_{d}$. According to Dominik and Decin (2003) the ISO observations can be explained by a simple model of collisional cascades, completed by some stirring in the case of high mass discs (cf. Kenyon \& Bromley 2002b). In a collisional cascade scenario where collisions occur with constant velocities, the decrease of the amount of dust can be described by a power law of index -1 and this applies to all observed debris discs. In the case of very low mass discs (about $10^{-3} M_{\oplus}$, practically unobserved so far), the slope of this power law can be of the order of -2 . In a scenario where the collisional cascade is continuously stirred (because the collision velocities increase with time), the power law slope can be steeper than -1 .

\section{Case studies}

A few stars were observed rather extensively and with several ISO observing modes or instruments because they were particularly interesting cases. The best examples are $\alpha$ Lyr (Vega), $\beta$ Pic, $\alpha$ PsA (Fomalhaut), $\rho^{1} \mathrm{CnC}$ and HD 207129.

\subsection{VEGA AND $\beta$ PIC}

The most detailed ISO studies of the discs around these two prototypes of debris discs are found in Heinrichsen et al. $(1998,1999)$. They are summarized in Table 1.

Based on the $25 \mu \mathrm{m}$ ISO data, the authors argue that the disc around $\beta$ Pic is in fact much more massive than the cool dust derived from the ISO $60 \mu \mathrm{m}$ emission. They suggest that there is some warm dust (300 to $500 \mathrm{~K}$ ), extension of the inner disc seen in the optical, and a significant amount of cool 
Table I. ISOPHOT results on Vega and $\beta$ Pic

\begin{tabular}{lll}
\hline ISOPHOT & Vega & $\beta$ Pic \\
\hline $\begin{array}{l}\text { High resolution } \\
\text { scans }\end{array}$ & $60 \mu \mathrm{m}(\mathrm{P} 32 / \mathrm{C} 100)$ & $25,60 \mu \mathrm{m}$ \\
Disc resolved & Yes: face-on & Yes: edge-on \\
Distance & $7.8 \mathrm{pc}$ & $19.3 \mathrm{pc}$ \\
Disc radius & $86 \mathrm{AU}$ at $60 \mu \mathrm{m}$ & $84 \mathrm{AU}$ at $25 \mu \mathrm{m}$ \\
& $140 \mathrm{AU}$ at $90 \mu \mathrm{m}$ & $140 \mathrm{AU}$ at $60 \mu \mathrm{m}$ \\
$\begin{array}{l}\text { Multifilter- } \\
\text { Photometry }\end{array}$ & $25,60,80,100,120$, & $4.85,7.3,11.3,12.8,16,25$ \\
$\begin{array}{l}\text { Adopted } \\
\text { dust emissivity }\end{array}$ & $Q(\lambda) \propto 1 / \lambda^{1.1}$ & $60,80,120,150 \& 170 \mu \mathrm{m}$ \\
$\begin{array}{l}\text { Dust mass } \\
\text { in disc }\end{array}$ & $1-5 \times 10^{-3} M_{\oplus}$ & $1.0-3.3 \times 10^{-2} M_{\oplus}$ \\
& Habing et al. $2001:$ & Habing et al. $2001:$ \\
& $1.3-13 \times 10^{-4} M_{\oplus}$ & $1.2-12 \times 10^{-2} M_{\oplus}$ \\
Reference & Heinrichsen et al.1998 & Heinrichsen et al.1999 \\
& Proposal: Walker & Proposal: Heinrichsen \\
\hline
\end{tabular}

dust in addition to that seen by ISO. The star could be surrounded by a large 'Oort' cloud of comets.

Walker \& Heinrichsen (2000) present ISOPHOT-S spectra (from 6 to $12 \mu \mathrm{m}$ ) of 12 Vega-like stars including the four 'prototypes' (Vega, $\beta$ Pic, Fomalhaut and $\varepsilon$ Eri) in search for silicates and PAHs features in debris discs. They found silicate dust emission towards two stars (HD 144432 and HD 139614), emission from carbon-rich molecules towards two others (HD 169142 and HD 34700) and emission from both towards HD 142666. For all their other stars, including the four 'prototypes', either only the photosphere is seen at these mid-infrared wavelengths, or some thermal featureless excess. The authors also present preliminary ISOPHOT maps at 60 and/or $90 \mu \mathrm{m}$, thus giving some insight in the extent and structure of the discs. The authors argue that the discs could be in the early stage of planet formation. However they give no accurate information on stellar age, hence the question: "Are the above 
features emitted by dust in a debris disc or in a much younger and thicker envelope or disc?".

\section{2. $\alpha$ PSA}

Fajardo-Acosta et al. (1997) have obtained P32 maps with ISOPHOT-C100 at $60 \mu \mathrm{m}$, with a spatial resolution of about $30^{\prime \prime}$ for six Vega-type systems. At $60 \mu \mathrm{m}$ there is no excess emission detected towards $\alpha \mathrm{CrB}, \sigma \mathrm{Her}$ or $\alpha \mathrm{Cen}$. There is a marginal detection of extended emission, out to $\sim 800 \mathrm{AU}$ or $30^{\prime \prime}$ from the star $\gamma \mathrm{Oph}$. Only $\alpha$ PsA in their sample shows a convincing excess emission in the range $\sim 30^{\prime \prime}$ to $80^{\prime \prime}$ i.e. $\sim 210$ to $560 \mathrm{AU}$, confirming the IRAS temperature of 58-75 $\mathrm{K}$ and suggesting grains up to $\sim 10 \mu \mathrm{m}$ in size. They estimate a dust mass of $\sim(2-6) \times 10^{-3} M_{\oplus}$. A comparison with $\beta$ Pic shows that both discs are similarly extended at $60 \mu \mathrm{m}$ (400 AU for $\beta$ Pic vs. 560 AU for $\alpha$ PsA).

\section{3. $\rho^{1} \mathrm{CNC}$}

The star $\rho^{1} \mathrm{CnC}$ is among the first 10 stars found to host a planet. In 1996, following the news of a planet around $\rho^{1} \mathrm{CnC}$, a special set of ISO observations was requested and carried out to search for a disc. The star $\rho^{1} \mathrm{CnC}$ was observed with ISOPHOT at $25 \mu \mathrm{m}$ (PHT03), and at 60, 90, 135 and $170 \mu \mathrm{m}$ (PHT22). Dominik et al. (1998) found an excess of $170 \pm 30 \mathrm{mJy}$ at $60 \mu \mathrm{m}$. They interpreted it as a debris disc located at about $60 \mathrm{AU}$ from the star and containing at least $4 \times 10^{-5} M_{\oplus}$ of dusty material. The star is a G8V, slightly metal-rich, located at a distance of $12.5 \mathrm{pc}$ from the Sun. From CaII H and K lines, its age was estimated to be 5 Gyr. Butler et al. (1997) detected the presence of a planet of period 14,65 days, implying a semi major axis of $0.11 \mathrm{AU}$. The inferred mass is $M_{2} \sin i=0.84 M_{\mathrm{Jup}}$. The far-infrared ISO excess was interpreted as a disc after any other possible origin had been examined and eleminated (companion M5, planet, cirrus knot in the background). The farinfrared spectrum of the excess is best fitted using cometary icy dust grains from the dust model by Li \& Greenberg (1997) as seen in Figure 2 of Dominik et al. (1998). These observations brought the first evidence of the coexistence of a disc and a planet around a star other than the Sun.

\subsection{HD 207129}

This solar-type (G2V) star is particularly interesting because of its cold debris disc, maybe one of the coldest observed so far. It caught the attention of Jourdain de Muizon et al. (1999) when they found a clear excess not only at $60 \mu \mathrm{m}$, but also at $170 \mu \mathrm{m}$ in their ISOPHOT-C200 data. Additional discretionary ISO observations were requested and obtained to get a more complete infrared spectrum of the disc. Based on the independent measurements of the 
CaII K line made by two groups of authors (Pasquini 1992 and Henry et al. 1996), Lachaume et al. (1999) determined a stellar age of 4.7 Gyr similar to that of the Sun, adopted by Jourdain de Muizon et al. (1999) and Habing et al. (2001). Zuckermann \& Webb (2000) argue that the star is a member of the Tucanae stream and must be as young as the Tucanae association (10$50 \mathrm{Myr}$ ) which is located at $\sim 45 \mathrm{pc}$. That is 3 times further than HD 207129 which is located at $15.6 \mathrm{pc}$ from the Sun, according to the Hipparcos Catalogue (Perryman et al. 1997). This significantly weakens the possibility of its belonging to the Tucanae stars. In any case the age of the star is less of an issue if it is young because it would then strengthen the case of Habing et al. $(1999,2001)$ that debris disc is the privilege of young stars (less than $400 \mathrm{Myr}$ ). HD 207129 is indeed one of the few puzzling exceptions in their study.

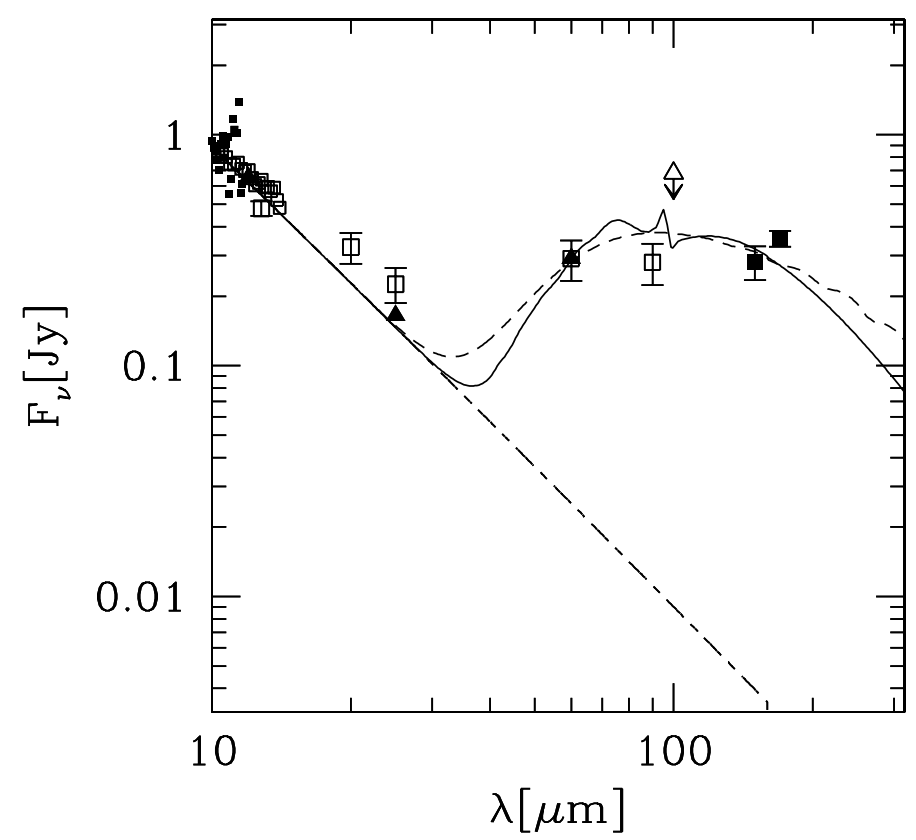

Figure 2. The infrared excess toward HD 207129. Comparison between two different dust compositions. The dashed line indicates a Draine \& Lee (1984) interstellar grain model, the solid line a Li \& Greenberg (1997) cometary dust model. The two cases differ most around $30-40 \mu \mathrm{m}$, but they both fit the remaining far-IR excess equally well (from: Jourdain de Muizon et al. 1999).

The infrared excess around HD 207129 is shown in Figure 2. The star emits approximately $1.1 \times 10^{-4}$ of its luminosity longwards of $25 \mu \mathrm{m}$. The excess emission is explained by assuming a disc of dust-like material equivalent to $2 \times 10^{-2} M_{\oplus}$. The dust temperature ranges from 10 to $50 \mathrm{~K}$, which makes it the coldest debris disc around a Vega-like star known to date. The dust distribution in the disc presents a circular hole at a distance of $\sim 400 \mathrm{AU}$ 
from the star. This hole should be filled within $10^{-3}$ of the stellar age by particles spiralling inward because of the Poynting-Robertson effect unless an agent sweeps it clean. It could thus be explained by the presence of one or more planets.

\section{Dust and gas composition of the dises}

Debris discs are essentially made of dust particles and larger bodies; however, these latter cannot be detected by ISO. In addition, the younger discs may still have a significant gas component. Before ISO, any attempt to trace the gas via $\mathrm{CO}$ molecular bands was unsuccessful. Direct measurement of $\mathrm{H}_{2}$ with ISO-SWS (de Graauw et al. 1996) has made a real break-through toward understanding the gas composition of very young discs.

\subsection{Dust COMPOSITION}

Amorphous silicates $(\mathrm{r}<1 \mu \mathrm{m})$ were detected in the ISOPHOT-S spectra of 9 classical T Tauri stars in the Chamaeleon I dark cloud (Natta et al. 2000). They discuss a model which explains the origin of this material in a hot, optically thin surface layer of a disc around the star, i.e in the disc atmosphere.

Crystalline silicates were detected by van den Ancker et al. (2001) toward the pre-main-sequence (B9.5Ve, A0II-IIIe or AOV ?) star $51 \mathrm{Oph}$, using SWS01 and LWS01 (Clegg et al. 1996; Gry et al., 2003) full scans. The solidstate bands and energy distribution indicate that the dust has formed recently; it is a very young disc, not a debris disc. Other emission bands from hot gas $(\sim 350 \mathrm{~K})$ such as $\mathrm{CO}, \mathrm{CO}_{2}, \mathrm{H}_{2} \mathrm{O}$ and $\mathrm{NO}$ dominate the $4-8 \mu \mathrm{m}$ spectrum. Both these gas and dust bands are unusual for a young star and are more typical of evolved AGB stars, although 51 Oph does not seem at all to belong to that class. The authors explore various possibilities for the nature of 51 Oph, among them a recent episode of mass loss from a Be star, the collision of two gas-rich planets and the accretion of a solid body as the star expands at the end of its short main-sequence life.

A variety of crystalline silicates, forsterite $\left(\mathrm{Mg}_{2} \mathrm{SiO}_{4}\right)$, has been found toward the star HD 100546. Malfait et al. (1998) present SWS01 and LWS01 full scans of this isolated Herbig Ae/Be star. Forsterite is present in the micrometeorites and interplanetary dust of our Solar System. The ISO spectrum of HD 100546 is very similar to that of comet Hale-Bopp published by Crovisier et al. (1997). The amount of forsterite in the disc of HD 100546 is equivalent to $10^{13}$ comets Hale-Bopp, strengthening the hypothesis by Grady et al. (1997) that the disc around HD 100546 contains a huge swarm of comets similar to the Oort cloud in the Solar System. Crystalline silicates have also been found in several other comets such as P/Halley. Malfait et al. (1998) 
argue that the crystallisation process occurs during the early phases of disc evolution around young stars.

The ISO-SWS and -LWS spectra of an additional 14 isolated Herbig Ae/Be stars are presented in Meeus et al. (2001). These stars are believed to be the more massive analogs of $\mathrm{T}$ Tauri stars; they are seen as the progenitors of Vega-like stars (Waters \& Waelkens 1998). Meeus et al. (2001) obtained a variety of mid-infrared spectra ranging from the amorphous silicates (as in the $\mathrm{M}$ supergiant $\mu \mathrm{Cep}$ ) to the crystalline silicates (as in comet Hale-Bopp). The variations in the shape of the $8-14 \mu \mathrm{m}$ part of the spectra indicate the prominence of one or the other form of silicates. Four out of the fourteen stars have no silicates. In most silicate stars in their sample, crystalline silicates are present; this is confirmed by the shape of the $15-28 \mu \mathrm{m}$ ISO-SWS spectra of the stars. PAH bands are also identified toward half of the stars, and all features are superposed on a near-IR to mid-IR continuum excess. The authors interpret the continuum in term of disc geometry: an optically thick, geometrically thin, power-law component and an optically thin flare region (black body component). Bouwman et al. (2001) got a further detailed insight into the $6-14 \mu \mathrm{m}$ spectrum of these stars in order to study the silicate grain processing. The $10 \mu \mathrm{m}$ silicate profile is modelled using three components: silica $\left(\mathrm{SiO}_{2}\right)$ responsible for the $8-9 \mu \mathrm{m}$ blue shoulder in the silicate band, forsterite contributing to the $11.3 \mu \mathrm{m}$ feature, and amorphous olivine with two typical grain sizes of 0.1 and $2.0 \mu \mathrm{m}$. They identify two causes for the observed shift in peak position of the silicate band: i) a change in average grain size from small $(0.1 \mu \mathrm{m})$ to big $(2 \mu \mathrm{m})$, as a result of the depletion of small grains in the inner region of the disc, due to coagulation and other effects, ii) a change in grain composition from amorphous silicate to a mixture of amorphous and $\mathrm{Mg}$-rich crystalline silicate (forsterite) which could be the result of thermal annealing in the inner regions of the disc. However crystallisation occurs on a longer timescale than coagulation. There is no correlation between dust composition and disc geometry.

\subsection{GAS COMPOSITION: $\mathrm{H}_{2}$}

Before ISO, any attempt to detect a gas component in Vega-like discs had been unsuccessful or inconclusive (see e.g. Liseau 1999). The classical method of tracing molecular hydrogen by observing $\mathrm{CO}$, widely applied in the interstellar medium, did not seem to help. Thi et al. (1999) discovered molecular $\mathrm{H}_{2}$ in the ISO-SWS spectra of the T Tauri star GG Tau. The detection of two pure rotational lines at 17.035 and $28.218 \mu \mathrm{m}$ provided a direct measure of the total amount of warm molecular gas in the disc around the star. The same group (Thi et al. 2001a, 2001b) found $\mathrm{H}_{2}$ lines in the SWS spectra of $4 \mathrm{~T}$ Tauri stars (spectral type $\mathrm{K}$ to $\mathrm{M}$ ), 7 Herbig Ae stars (spectral type A to F), and 3 main-sequence stars including $\beta$ Pic (spectral type A to F). Their 
data suggest the presence of warm gas $(\mathrm{T} \approx 100-200 \mathrm{~K})$, which mass ranges from $\sim 10^{-4} M_{\odot}$ up to $8 \times 10^{-3} M_{\odot}$, around all the stars they observed. This mass corresponds to $1 \%-10 \%$ of the total disc mass inferred from millimeter continuum observations, and a much higher fraction in the case of debris discs. Additional $\mathrm{CO}$ observations show that $\mathrm{CO}$ is not a good tracer of the gas in circumstellar discs. The amount of $\mathrm{CO}$ gas is likely strongly affected by photodissociation due to the stellar and interstellar ultraviolet radiation in the surface layers of the disc and freeze-out onto grain surface in the midplane. Direct measurement of $\mathrm{H}_{2}$ leads to a gas-to-dust ratio of $\sim 100$ in the debris discs of $\beta$ Pic and HD 135344, similar to the interstellar medium ratio. The bulk of the gas around pre-main-sequence stars is cool $(\mathrm{T} \approx 20-80 \mathrm{~K})$, while the warm gas $(\mathrm{T} \approx 100-200 \mathrm{~K})$ may constitute the major gaseous component of debris discs around main-sequence stars, thus providing a reservoir for the formation of Jovian planets.

\section{Conclusion and open questions}

The ISO Data Archive has not yet been fully exploited as far as debris discs and their precursors are concerned. Several aspects of disc evolution are not well understood. Why and how do most discs disappear after a lifetime of about 400 Myr ? Why do some stars keep a disc for much longer, up to several Gyr ? Do discs coexist with planets ? Is it systematic, and if not, what conditions lead to a disc-planets or disc-only system ? Does it depend on the stellar spectral type ? Which physical and chemical processes occur during the evolution of a disc ? Although the ISO data has already provided partial answers to some of these questions, it is clear that our picture is still incomplete. Beyond the IDA, space projects such as Spitzer and the Herschel Space Observatory do and will follow up ISO observations of debris discs. Spitzer has a similar wavelength range as ISO but a higher sensitivity; it is able to probe deeper and, hence, perform similar kind of statistics as ISO but on a larger volume around the Sun, thus expected to detect photometrically hundreds of debris discs. Some preliminary Spitzer results on the few brightest discs also address the structure of the discs, dust grain composition, and search for substellar companions. Herschel will open a new window since it extends to the submillimeter range. This will allow to trace the cold component of the discs such as the dust located in the Kuiper Belt, the Oort cloud and still further from the star. 


\section{References}

Aumann, H.H., F.C. Gillett, C.A. Beichman, T. de Jong, J.R. Houck, F.J. Low, G. Neugebauer, R.G. Walker, and P.R. Wesselius. Discovery of a shell around Alpha Lyrae. ApJ 278, L23, 1984.

Backman, D. and F. Paresce. Main-sequence stars with circumstellar solid material - The VEGA phenomenon. In E. Levy and J. Lunine, editors, Protostars and Planets III, pp.1253-1304. Tucson: University of Arizona press, 1993.

Bouwman, J., G. Meeus, A. de Koter, S. Hony, C. Dominik and L.B.F.M. Waters. Processing of silicate dust grains in Herbig Ae/Be systems. A\&A 375, 950, 2001.

Butler, R.P., G.W. Marcy, E. Williams, H. Hause and P. Shirts. Three New "51 Pegasi-Type" Planets. ApJ 474, L115,1997.

Clegg, P.E. et al. 1996, A\&A 315, L38

Crovisier, J., K. Leech, D. Bockelee-Morvan, T.Y. Brooke, M.S. Hanner, B. Altieri, H.U. Keller and E. Lellouch. The spectrum of Comet Hale-Bopp (C/1995 01) observed with the Infrared Space Observatory at 2.9 AU from the Sun. Science 275, 1904, 1997.

Decin, G., C. Dominik, K. Malfait, M. Mayor and C. Waelkens. The Vega phenomenon around G dwarfs. A\&A 357, 533, 2000,.

Decin, G., C. Dominik, L. B. F. M. Waters and C. Waelkens. Age Dependence of the Vega Phenomenon: Observations. A\&A 598, 636, 2003.

de Graauw, Th., L.N. Haser, D.A. Beintema, P.R. Roelfsema and 58 authors. Observing with the ISO Short-Wavelength Spectrometer. A\&A 315, L49,1996.

Dominik, C., R.J. Laureijs., M. Jourdain de Muizon and H.J. Habing. A Vega-like disk associated with the planetary system of rho (1) CNC. A\&A 329, L53, 1998.

Dominik, C. and G. Decin. Age Dependence of the Vega Phenomenon: Theory. A\&A 598, 626, 2003.

Draine, B.T. and H.M. Lee. Optical properties of interstellar graphite and silicate grains. ApJ 285, 89, 1984.

Fajardo-Acosta, S.B., R.E. Stencel and D.E. Backman. Infrared Space Observatory Mapping of $60 \mu \mathrm{mDust}$ Emission Around Vega-type Systems. ApJ 487, L151, 1997.

Fajardo- Acosta, S.B., R.E. Stencel, D.E. Backman and N. Thakur. Infrared Space Observatory Photometric Search of Main-Sequence Stars for Vega-Type Systems. ApJ 520, 215, 1999.

Grady, C.A., M.L. Sitko, K.S. Bjorkman, M.R. Perez, D.K. Lynch, R.W. Russell and M.S. Hanner. The Star-grazing Extrasolar Comets in the HD 100546 System. ApJ 483, 449, 1997.

Greaves, J.S. and M.C. Wyatt. Some anomalies in the occurrence of debris discs around main-sequence A and G stars. MNRAS 345, 1212, 2003.

Habing, H.J., C. Dominik, M. Jourdain de Muizon, M.F. Kessler, R.J. Laureijs, K. Leech, L. Metcalfe, A. Salama, R. Siebenmorgen and N. Trams. Disappearance of stellar debris disks around main-sequence stars after 400 million years. Nature 401, 456, 1999.

Habing, H.J., C. Dominik, M. Jourdain de Muizon, R.L. Laureijs, M.F. Kessler, K. Leech, L. Metcalfe, A. Salama, R. Siebenmorgen, N. Trams and P. Bouchet. Incidence and survival of remnant disks around main-sequence stars. A\&A 365, 545, 2001.

Heinrichsen, I. , H.J. Walker and U. Klaas. Infrared mapping of the dust disc around Vega. MNRAS 293, L78, 1998.

Heinrichsen, I., H.J. Walker, U. Klaas, R.J. Sylvester and D. Lemke. An infrared image of the dust disc around beta Pic. MNRAS 304, 589, 1999.

Henry, T.J., D.R. Soderblom, R.A. Donahue and S.L. Baliunas. A Survey of Ca II H and K Chromospheric Emission in Southern Solar-Type Stars. AJ 111, 439, 1996.

Johnson, H.M. and C.D. Wright . Predicted Infrared Brightness of Stars within 25 parsecs of the Sun. ApJS 53, 643, 1983. 
Jourdain de Muizon, M., R.J. Laureijs, C. Dominik, H.J. Habing, L. Metcalfe, R. Siebenmorgen, M.F. Kessler, P. Bouchet, A. Salama, K. Leech, N. Trams, and A. Heske. A very cold disc of dust around the G0V star HD 207129. A\&A 350, 875, 1999.

Jourdain de Muizon, M., R.J. Laureijs, H.J. Habing, K. Leech., M.F. Kessler, L. Metcalfe, A. Salama, R. Siebenmorgen, C. Dominik, N. Trams and P. Bouchet. The Moon and Extra-Solar Planets. Earth, Moon and Planets 85-86, 201, 2001.

Jourdain de Muizon, M. and R.J. Laureijs. Debris disks around main- sequence stars with ISO: 60 and 90 micron observations. A\&A in preparation, 2005.

Kenyon, S.J. and B.C. Bromley. Collisional Cascades in Planetesimal Disks. I. Stellar Flybys. AJ 123, 1757, 2002a.

Kenyon, S.J. and B.C. Bromley. Dusty Rings: Signposts of Recent Planet Formation. ApJ 577, L35, 2002b.

Kessler, M.F., J.A. Steinz, M.E. Anderegg, J. Clavel, G. Drechsel, P. Estaria, J. Faelker, J.R. Riedinger, A. Robson, B.G. Taylor and S. Ximénez de Ferrán. The Infrared Space Observatory (ISO) mission. A\&A 315, L27, 1996.

Kessler, M.F., Mller, T.G., Leech, K. et al. 2003, 'The ISO Handbook, Volume I: ISO - Mission \& Satellite Overview', ESA SP-1262

Lachaume, R., C. Dominik, T. Lanz and H.J. Habing. Age determinations of main-sequence stars: combining different methods. A\&A 348, 897, 1999.

Lagrange, A.-M., D. Backman and P. Artymowicz. Planetary Material around Main-Sequence Stars. In V. Mannings, A.P. Boss and S.S. Russell, editors, Protostars and Planets IV, p. 639. Tucson: University of Arizona Press, 2000.

Laureijs, R.J., M. Jourdain de Muizon, K. Leech, R. Siebenmorgen, C. Dominik, H.J. Habing, N. Trams and M.F. Kessler. A 25 micron search for Vega- like disks around main-sequence stars with ISO. A\&A 387, 285, 2002.

Laureijs, R.J., Klaas, U., Richards, P.J. et al. 2003, 'The ISO Handbook, Volume IV: PHT The Imaging Photo-Polarimeter', ESA SP-1262

Leech, K., Kester, D., Shipman, R. et al. 2003 ESA SP-1262

Lemke, D., U. Klaas, J. Abolins, P. Ábrahám and 45 authors ISOPHOT - capabilities and performance. A\&A 315, L64, 1996.

Li, A. and J.M. Greenberg. A unified model of interstellar dust. A\&A 323, 566, 1997.

Liseau, R. Molecular line observations of southern main-sequence stars with dust disks: alpha Ps A, beta Pic, epsilon Eri and HR 4796 A. Does the low gas content of the beta Pic and varepsilon Eri disks hint of planets? A\&A 348, 133, 1999.

Malfait, K., C. Waelkens, L.B.F.M. Waters, B. Vandenbussche, E. Huygen and M.S. de Graauw. The spectrum of the young star HD 100546 observed with the Infrared Space Observatory. A\&A 332, L25, 1998.

Meeus, G., L.B.F.M. Waters, J. Bouwman, M.E. van den Ancker, C. Waelkens and K. Malfait. ISO spectroscopy of circumstellar dust in 14 Herbig Ae/Be systems: Towards an understanding of dust processing. A\&A 365, 476, 2001.

Morbidelli, A., J.-M. Petit, B. Gladman and J. Chambers. A plausible cause of the late heavy bombardment. Meteorit. Planet. Sci., 36, 371, 2001.

Natta, A., M.R. Meyer, S.V.W. Beckwith. Silicate Emission in T Tauri Stars: Evidence for Disk Atmospheres? ApJ 534, 838, 2000.

Pasquini, L. The Ca II K line in solar type stars. A\&A 266, 347, 1992.

Perryman, M.A.C., L. Lindegren, J. Kovalevsky and 16 authors. The Hipparcos Catalogue. A\&A 323, L49, 1997.

Perryman, M.A.C. et al. The Hipparcos and Tycho catalogues. Astrometric and photometric star catalogues derived from the ESA Hipparcos Space Astrometry Mission, Publisher: Noordwijk, Netherlands: ESA Publications Division, Series: ESA SP Series vol no: 1200, ISBN: 9290923997 (set), 1997. 
Plets, H. and C. Vynckier. An analysis of the incidence of the Vega phenomenon among main-sequence and post main-sequence stars. A\&A 343, 496, 1999.

Robberto, M., M.R. Meyer, A. Natta, S.V.W. Beckwith. ISOPHOT observations of circumstellar disks around young stellar objects. In P. Cox and M.F. Kessler, editors, The Universe as seen by ISO, ESA SP-427, 195, 1999.

Shoemaker E. and C. Shoemaker. The Role of Collisions. In J.K. Beatty, C. Petersen and A. Chaikin, editors, The New Solar System 4th edition, eds, Sky Publishing Corporation and Cambridge University Press, 69, 1999.

Spangler, C., A.I. Sargent, M.D. Silverstone, E.E. Becklin and B. Zuckerman. Dusty Debris around Solar-Type Stars: Temporal Disk Evolution. ApJ 555, 932, 2001.

Thi, W.F., E.F. van Dishoeck, G.A. Blake, G.J. van Zadelhoff and M.R. Hogerheijde. Detection of $\mathrm{H}_{2}$ Pure Rotational Line Emission from the GG Tauri Binary System. ApJ 521, L63, 1999.

Thi, W.F., G.A. Blake, E.F. van Dishoeck, G.J. van Zadelhoff, J.M.M. Horn, E.E. Becklin, V. Mannings, A.I. Sargent, M.E. van den Ancker and A. Natta. Substantial reservoirs of molecular hydrogen in the debris disks around young stars. Nature 409, 60, $2001 \mathrm{a}$.

Thi, W.F., E.F. van Dishoeck, G.A. Blake, G.J. van Zadelhoff, J.M.M. Horn, E.E. Becklin, V. Mannings, A.I. Sargent, M.E. van den Ancker, A. Natta and J. Kessler. $\mathrm{H}_{2}$ and CO Emission from Disks around T Tauri and Herbig Ae Pre-Main-Sequence Stars and from Debris Disks around Young Stars: Warm and Cold Circumstellar Gas. ApJ 561, 1074, 2001b.

van den Ancker, M.E., G. Meeus, J. Cami, L.B.F.M. Waters and C. Waelkens. The composition of circumstellar gas and dust in 51 Oph. A\&A 369, L17, 2001.

Walker, H.J. and I. Heinrichsen. ISOPHOT Observations of Dust Disks around Main Sequence (Vega-Like) Stars. Icarus 143, 147, 2000.

Waters, L.B.F.M. and C. Waelkens. Herbig Ae/Be Stars. ARA\&A 36, 233, 1998.

Zuckerman, B. and R.A. Webb. Identification of a Nearby Stellar Association in the Hipparcos Catalog: Implications for Recent, Local Star Formation. ApJ 535, 959, 2000.

Zuckerman, B. Dusty Circumstellar Disks. ARA\&A 39, 549, 2001. 